\title{
Divergent relationship of circulating CTRP3 levels between obesity and gender: A cross-sectional study
}

\author{
Roy Marshal Wagner ${ }^{1}$, Kamesh Sivagnanam ${ }^{2}$, William Andrew Clark ${ }^{1}$, Jonathan M Peterson Corresp. 3,4 \\ 1 Allied Health Sciences, College of Clinical and Rehabilitative Health Sciences, East Tennessee State University, Johnson City, TN, United States \\ 2 Department of Internal Medicine, Quillen College of Medicine, East Tennessee State University, Johnson City, TN, United States \\ 3 Department of Biomedical Sciences, Quillen College of Medicine, East Tennessee State University, Johnson City, TN, United States \\ 4 Department of Health Sciences, East Tennessee State University, Johnson City, TN, USA \\ Corresponding Author: Jonathan M Peterson \\ Email address: petersonjm1@etsu.edu
}

Clq TNF Related Protein 3 (CTRP3) is a novel adipose tissue derived secreted factor, or adipokine, which has been linked to a number of beneficial biological effects on metabolism, inflammation, and survival signaling in a variety of tissues. However, very little is known about CTRP3 in regards to human health. The purpose of this project was to examine circulating CTRP3 levels in a clinical population, patients with symptoms requiring heart catheterization in order to identify the presence of obstructive coronary artery disease (CAD). It was hypothesized that serum CTRP3 levels would be decreased in the presence of CAD. Methods: Body mass index (BMI), diabetes status, and plasma samples were collected from 100 patients who were $>30$ years of age and presented at the East Tennessee State University Heart Clinic with symptoms requiring heart catheterization in order to identify the presence of cardiovascular blockages ( $n=52$ male, $n=48$ female). Circulating CTRP3 levels were quantified using commercially available ELISA. Results: Circulating CTRP3 levels had no relationship to the presence of CAD regardless of gender. However, circulating concentrations of CTRP3 were significantly higher in normal weight (BMI <30) females $(0.88+/-0.12 \mu \mathrm{g} / \mathrm{ml})$ compared with males $(0.54+/-0.06 \mu \mathrm{g} / \mathrm{ml})$. Further, obesity $(\mathrm{BMI}>30)$ resulted in an increase in circulating CTRP3 levels in male subjects $(0.74+/-0.08 \mu \mathrm{g} / \mathrm{ml})$ but showed a significant decrease in female subjects $(0.58$ $+/-0.07 \mu \mathrm{g} / \mathrm{ml}$ ). Additionally, there was a significant reduction in circulating CTRP3 levels in female subjects who were diagnosed with Type 2 diabetes compared with patients without $(0.79+/-0.08$ vs. $0.42+/-0.10 \mu \mathrm{g} / \mathrm{ml})$. There was no relationship between diabetes status and circulating CTRP3 levels in male subjects. Conclusion: Circulating CTRP3 levels had a different relationship with diabetes and obesity status between male and female patients. It is possible that circulating CTRP3 levels are controlled by hormonal status, however more research is needed to explore this relationship. Nevertheless, future studies examining the relationship between CTRP3 levels and disease status should treat 
gender as an independent variable. 
1 Divergent relationship of circulating CTRP3 levels between obesity and gender: A Cross-

2

3

4 R. Marshal Wagner ${ }^{1,5}$, Kamesh Sivagnanam ${ }^{2}$, W. Andrew Clark ${ }^{1}$, and Jonathan M. Peterson ${ }^{3,4^{*}}$

5

6 1: Allied Health Sciences, College of Clinical and Rehabilitative Health Sciences, East

7 Tennessee State University, Johnson City, Tennessee

8

9

10

11

12

13

14

15

16

17

18

19

20

21

22

23

24

2: Department of Internal Medicine, Quillen College of Medicine, East Tennessee State University, Johnson City, Tennessee

3:College of Public Health, Department of Health Sciences, East Tennessee State University, Johnson City, Tennessee

4: Quillen College of Medicine, Department of Biomedical Sciences, East Tennessee State University, Johnson City, Tennessee

5: Undergraduate student.

*Corresponding author

Email: petersonjm1@etsu.edu (JMP) 


\section{ABSTRACT:}

C1q TNF Related Protein 3 (CTRP3) is a novel adipose tissue derived secreted factor, or adipokine, which has been linked to a number of beneficial biological effects on metabolism, inflammation, and survival signaling in a variety of tissues. However, very little is known about CTRP3 in regards to human health. The purpose of this project was to examine circulating CTRP3 levels in a clinical population, patients with symptoms requiring heart catheterization, in order to identify the presence of obstructive coronary artery disease (CAD). It was hypothesized that serum CTRP3 levels would be decreased in the presence of CAD. Methods: Body mass index (BMI), diabetes status, and plasma samples were collected from 100 patients who were $>30$ years of age and presented at the East Tennessee State University Heart Clinic with symptoms requiring heart catheterization in order to identify the presence of cardiovascular blockages $(n=52$ male, $n=48$ female). Circulating CTRP3 levels were quantified using commercially available ELISA. Results: Circulating CTRP3 levels had no relationship to the presence of CAD regardless of gender. However, circulating concentrations of CTRP3 were significantly higher in normal weight (BMI <30) females $(0.88 \pm 0.12 \mu \mathrm{g} / \mathrm{ml})$ compared with males $(0.54 \pm 0.06 \mu \mathrm{g} / \mathrm{ml})$. Further, obesity $(\mathrm{BMI}>30)$ resulted in an increase in circulating CTRP3 levels in male subjects $(0.74+0.08 \mu \mathrm{g} / \mathrm{ml})$ but showed a significant decrease in female subjects $(0.58 \pm 0.07 \mu \mathrm{g} / \mathrm{ml})$. Additionally, there was a significant reduction in circulating CTRP3 levels in female subjects who were diagnosed with Type 2 diabetes compared with patients without $(0.79 \pm 0.08$ vs. $0.42+0.10 \mu \mathrm{g} / \mathrm{ml})$. There was no relationship between diabetes status and circulating CTRP3 levels in male subjects. Conclusion: Circulating CTRP3 levels had a different relationship with diabetes and obesity status between male and female patients. It is possible that circulating CTRP3 levels are controlled by hormonal status, however more research is needed to explore this relationship. Nevertheless, future studies 
48 examining the relationship between CTRP3 levels and disease status should treat gender as an 49 independent variable. 
50

51

52

53

54

55

56

57

58

59

60

61

62

63

64

65

66

67

68

69

70

71

\section{INTRODUCTION:}

Cardiovascular disease is the leading cause of death worldwide (Gaziano et al. 2006). Recent studies have shown that obesity and diabetes mellitus are two of the largest risk factors for the development of coronary heart disease, even more so than cigarette smoking, dyslipidemia, and even hypertension (Smith 2007). Obesity is a chronic disease affecting over one-third of US adults (Ben-Menachem 2007; Flegal et al. 2012; Ogden et al. 2012). However, the mechanisms by which obesity and diabetes mellitus increase the prevalence of cardiovascular disease are still being actively investigated.

Dysfunctional adipose tissue represents a novel paradigm that mechanistically links obesity to a variety of diseases such as cardiovascular disease. Traditionally adipose tissue is thought of as solely a storage vessel for excess lipids; however, adipose tissue secretes a number of factors, which have essential endocrine roles in regulating biological functions (Garg 2006; Jankovic et al. 1969; Wang et al. 1986). These adipose tissue-derived secreted factors are collectively called adipokines. Efforts to identify novel adipokines have led to the discovery of a family of secreted proteins, designated as C1q TNF-related protein 1-15 (CTRP1-15) (Peterson et al. 2010; Seldin et al. 2012; Seldin et al. 2014; Wei et al. 2012; Wei et al. 2011; Wei et al. 2013; Wong et al. 2009; Wong et al. 2008). The CTRP proteins, adiponectin, TNF-alpha, as well as other proteins with the $\mathrm{C} 1 \mathrm{q}$ domain together are grouped together as the C1q/TNF superfamily (Wong et al. 2004). Of these proteins CTRP3 (synonyms CORS-26, cartducin and cartonectin) has been demonstrated to protect against ischemia-induced cardiac damage (Wu et al. 2015; Yi et al. 2012) and circulating CTRP3 levels were recently found to be reduced in patients with acute coronary syndrome (Choi et al. 2014). 
72 Previous research has shown that circulating CTRP3 levels are reduced with conditions associated

73 with cardiovascular events such as obesity and metabolic syndrome (Ban et al. 2014; Deng et al.

74 2015; Qu et al. 2015; Wolf et al. 2015). Conversely, CTRP3 levels have also be reported to be

75 elevated with obesity and/or metabolic syndrome (Choi et al. 2012). Regardless, the potential use

76 of circulating CTRP3 levels as a biomarker for risk or the presence of cardiovascular disease has

77 not been established. These data led to our initial hypothesis: patients with obstructive coronary

78 artery disease (CAD) will have reduced circulating CTRP3 levels compared with patients without

79 CAD. Although our data did not support our initial hypothesis, we did find a number of novel

80 observations regarding the relationship of circulating CTRP3 levels, gender and obesity that sheds

81 light on some of the contradictory reports of the CTRP3 in the literature.

82

83

84

85

86

87

88

89

90

91

92

93

94

\section{METHODS}

\section{Ethics Statement}

All study subjects provided written informed consent for the study procedures. The study was approved by the Institutional Review Board of East Tennessee State University's Office of Research and Sponsored Programs (IRB \#0313-35s, 2013, ETSU/VA Medical IRB Board).

\section{Study Design and Participants}

This was a cross-sectional study conducted from June 2013 to June 2015 at the East Tennessee State University Heart Clinic, an affiliate of James H Quillen College of Medicine Division of Cardiology. Inclusion criteria included: Patients with symptoms requiring heart catheterization in order to identify the presence of obstructive coronary artery disease (CAD). Exclusion criteria included known history of $\mathrm{CAD}$, Coronary artery bypass grafting (CABG), coronary stent placement, type 1 diabetes and/or $<30$ years of age. 
95

96

97

\section{Clinical and Laboratory Measurements}

Self-reported measures of age, sex, ethnicity, and smoking status were obtained from each participant. Clinical data (diagnosis of Type 2 Diabetes, Blood pressure, height, and weight) were obtained as part of routine medical examination. Body mass index (BMI) was calculated as weight/height ${ }^{2}\left(\mathrm{~kg} / \mathrm{m}^{2}\right)$. Blood samples for lipid analysis were obtained through a commercial lab as part of the routine medical visit. For plasma collection samples were collected from non-fasting trial participants into 5-mL EDTA vacutainers, immediately refrigerated, transported to the laboratory, and processed within 24 hours after withdrawal. Samples were centrifuged at room temperature for 10 minutes at $3000 \mathrm{x}$ g, the plasma (supernatant) was decanted using a Pasteur pipette and transferred to a $3 \mathrm{~mL}$ glass amber vial and stored at $-30^{\circ} \mathrm{C}$ until further analysis. Human CTRP3 levels were measured by ELISA (Aviscera Bioscience Cat\#SK00082) according to manufacturer's protocol, with an intra-assay coefficient of variation of less than $10 \%$. Samples with CTRP3 levels below the detection limit of the assay were assigned the lowest detectable value $(0.077 \mu \mathrm{g} / \mathrm{ml})$. Glucose levels were measured by commercial glucose analyzer (One touch Ultra).

\section{Statistical Analysis}

Frequencies, means, and standard deviations were calculated for descriptive analyses (Table 1). Our analysis considered gender as an independent variable and a 2-way ANOVA was performed for categorical variables (presence of blockage, diabetes, obesity (BMI >30), smoker) with Fisher's LSD test analysis for multiple comparisons. Spearman's correlations were perform for continuous variables. Significance was set at $\mathrm{p} \leq 0.05$ and a statistical trend was defined as $0.05<\mathrm{p} \leq 0.09$. Statistical analyses were conducted using GraphPad 6.0. 


\section{RESULTS AND DISCUSSION}

\section{Study Population}

118 There were a total of 100 participants ( $n=52$ male, $n=48$ female), 28 were positive for CAD. The 119 remaining 72 participants did not have significant findings during catheterization. The average age 120 of the study subjects was $57.5 \pm 12.6$ years, and their average BMI was $33.09 \pm 7.9 \mathrm{~kg} / \mathrm{m}^{2}$. 121 Additional descriptive statistics are listed in Table 1.

Table 1: Categorical population Overview

\begin{tabular}{r|lll}
\hline \multicolumn{1}{c}{ Overall } & Male & Female \\
\hline Total & 100 & 52 & 48 \\
Obese (BMI >30) & 57 & 24 & 33 \\
Type 2 Diabetes & 30 & 15 & 15 \\
Smoker & 53 & 30 & 20 \\
Presence of CAD & 29 & 18 & 10 \\
\hline
\end{tabular}

\section{Relationship between CTRP3 and Categorical variables}

125

126

127

128

129

130

131

132

In this cross-sectional study of patients with a variety of symptoms, we initially hypothesized that because CTRP3 has cardioprotective properties lower circulating CTRP3 levels would be associated with CAD. Associations between circulating CTRP3 levels and anthropomorphic measurements, presence of diabetes, gender, and obesity were also investigated. No differences were observed in this population regarding circulating CTRP3 levels and CAD, dyslipidemia, hypertension, or tobacco use (figure 1). There was not a significant difference in circulating CTRP3 levels between male and female subjects (figure 2A), however CTRP3 levels were significantly elevated in lean females compared with lean male subjects (figure 2B). Further, 
133 circulating CTRP3 levels were significantly higher in obese compared with lean males and

134 conversely, CTRP3 levels were significantly lower in obese females compared with lean females

135 (Figure 2B). While the presence of diabetes had no association with circulating CTRP3 levels in

136 male subjects, diabetic females had close to a 50\% lower level of circulating CTRP3 (figure 2C).

137 Although this study was limited to this specific clinical subgroup (those with symptoms requiring

138 catheterization), there were a number of interesting and novel observations made that shed light

139 on the conflicting findings of the relationship between diabetes and obesity have with circulating

140 CTRP3 levels (Ban et al. 2014; Choi et al. 2012; Deng et al. 2015; Qu et al. 2015; Wolf et al. 141 2015).

142 Relationship between CTRP3 and Continuous variables

143 We examined the relationship between circulating CTRP3 levels and age, cholesterol,

144 triglycerides, glucose, and BMI across the population and did not observe significant relationships

145 (table 2). We then repeated the analysis independently with each gender and observed that in the

146 males there was a significant positive correlation between BMI and circulating CTRP3 levels in

147 males (table 3).

Table 2: Overall Relationship to Circulating CTRP3 levels

\begin{tabular}{llllll}
\hline & P-value & r-value & Mean & & SD \\
\hline AGE (yrs) & 0.089 & -0.137 & 57.46 & \pm & 12.66 \\
Total Cholesterol (mg/dL) & 0.123 & -0.131 & 171.60 & \pm & 43.19 \\
HDL (mg/dL) & 0.111 & -0.137 & 39.35 & \pm & 9.66 \\
LDL (mg/dL) & 0.069 & -0.169 & 100.40 & \pm & 42.00 \\
TRIGLY (mg/dL) & 0.430 & -0.020 & 163.50 & \pm & 88.01 \\
Glucose (mg/dL) & 0.178 & 0.094 & 90.65 & \pm & 53.38 \\
BMI & 0.276 & 0.061 & 33.09 & \pm & 7.89 \\
\hline
\end{tabular}

149

Table 3: Male Relationship to Circulating CTRP3 levels

\begin{tabular}{llllll}
\hline & P-value & r-value & Mean & SD \\
\hline AGE (yrs) & 0.281 & -0.084 & 56.06 & \pm 13.6 \\
Total Cholesterol (mg/dL) & 0.166 & -0.157 & 160.10 & \pm 39.6
\end{tabular}




\begin{tabular}{lllll} 
HDL $(\mathrm{mg} / \mathrm{dL})$ & 0.134 & -0.180 & 36.00 & \pm 8.3 \\
LDL $(\mathrm{mg} / \mathrm{dL})$ & 0.168 & -0.158 & 89.21 & \pm 39.6 \\
TRIGLY (mg/dL) & 0.351 & -0.063 & 169.1 & \pm 86.4 \\
Glucose $(\mathrm{mg} / \mathrm{dL})$ & 0.354 & 0.054 & 90.00 & \pm 54.2 \\
BMI & $0.020^{*}$ & 0.291 & 31.73 & \pm 7.5 \\
\hline
\end{tabular}

151

152 
Table 4: Female Relationship to Circulating CTRP3 levels

\begin{tabular}{lccrl}
\hline & P-value & r-value & \multicolumn{1}{c}{ Mean } & SD \\
\hline AGE (yrs) & 0.130 & -0.165 & 58.42 & \pm 11.40 \\
Total Cholesterol (mg/dL) & 0.193 & -0.141 & 184.40 & \pm 43.39 \\
HDL (mg/dL) & 0.193 & -0.141 & 42.85 & \pm 9.82 \\
LDL (mg/dL) & 0.289 & -0.092 & 112.30 & \pm 41.93 \\
TRIGLY (mg/dL) & 0.263 & 0.103 & 160.40 & \pm 90.18 \\
Glucose (mg/dL) & 0.252 & 0.099 & 91.13 & \pm 53.31 \\
BMI & 0.215 & -0.116 & 34.42 & \pm 8.22 \\
\hline
\end{tabular}

154

155 Our initial analysis found few overall correlations between circulating CTRP3 levels and any of

156 the variables listed. Although disappointing a thorough examination of the literature highlighted

157 that circulating CTRP3 levels are higher in females than males (Choi et al. 2012; Peterson et al.

158 2010; Wolf et al. 2015). This observation combined with new NIH guidelines for considering sex

159 as a biological variable caused us to reexamine our data using gender as an independent variable.

160 After this analysis we observed a number of significant differences between the gender regarding

161 circulating CTRP3 levels and obesity, dislipidemia, and Type 2 diabetes. To the best of our

162 knowledge this is the first study to independently examine CTRP3 levels by both gender and

163 obesity. Although it has been previously reported that circulating CTRP3 levels are higher in

164 females than males, our finding of a divergent association of between the genders and obesity,

165 diabetes, and dislipidemia are completely novel observations. Furthermore, these observations

166 shed light on some of the discrepancy in the literature. For example, Wolf et al. (Wolf et al. 2015)

167 measured circulating CTRP3 levels in lean and obese subjects and showed CTRP3 levels were

168 reduced with obesity. However, $74 \%$ of the study population and $89 \%$ of the obese subjects were

169 female, therefore our findings are consistent in that obese females have lowers circulating CTRP3

170 levels. Choi et al. (Choi et al. 2014) reported no significant association between CTRP3 levels and

171 BMI in a study with a higher proportion of males (244/118 male/female) but did report negative 
172 association with CTRP3 and BMI in another study of 349 subjects (134/215 male/female) (Choi

173 et al. 2012). However, in both manuscripts the authors analyzed the association between CTRP3

174 and BMI across the population and the opposing trajectories of gender as a factor potentially

175 masked the discovery of significant associations. Direct query to the authors for clarification,

176 confirmed that significant differences in CTRP3 levels were observed between the genders (Choi

177 et al., personal communications).

178 We found that in female subjects the presence of diabetes was associated with lower circulating

179 CTRP3 levels. Interestingly, this observation is supported by the work of Tan et al. who reported 180 that women with polycystic ovary syndrome had lower levels of CTRP3 than control subjects (Tan 181 et al. 2013). Polycystic ovary syndrome is an endocrine system disorder associated with obesity, 182 diabetes, dyslipidemia, and cardiovascular complications. Further, in this population CTRP3 levels 183 were restored with Metformin treatment, which is the most common drug used to treat type 2 184 diabetes (Tan et al. 2013). On the other hand Choi et al. examined circulating CTRP3 levels in 185 partially female dominated subject population (349 subjects; 134/215 male/female) and reported 186 that CTRP3 levels were increased in patients with type 2 diabetes (Choi et al. 2012). This finding 187 is contradictory to our findings and highlights that there is still much unknown regarding the 188 regulation of circulating CTRP3 levels. Further, although authors reported that women had a $\sim 25 \%$ 189 higher level of CTRP3 than men (Choi et al. 2012) they did not analyze the relationship between 190 obesity and CTRP3 levels independently by gender.

191 CTRP3 is thought to improve insulin sensitivity and metabolic function, particularly in the liver 192 (Peterson et al. 2010). Additionally, CTRP3 has been shown to reduce myocardial damage 193 following ischemia (Wu et al. 2015; Yi et al. 2012). Currently there are no clinically defined 194 biological cutoffs for circulating CTRP3 levels, however, our work indicates that future studies 
195 regarding the clinical significance of CTRP3 must evaluate difference between the genders

196 separately as the effect of metabolic dysfunction (obesity and/or diabetes) has conflicting effect

197 on circulating CTRP3 levels between the genders.

\section{Study limitations and conclusion}

199 One limitation of this study is that it comprised of only a clinical cardiovascular population

200 recruited from a single region and our observations may not apply to the general population. The

201 second and primary limitation of this study is that we were only able to obtain non-fasting samples

202 from the study population, which significantly increased the variability of the data and our results

203 may not have been observed in a fasting population. This is especially important to note

204 considering that, unlike many adipokines, CTRP3 levels have been reported to increase with

205 fasting (Peterson et al. 2010). Further, the type and sensitivity of the antibodies used in the ELISA,

206 may also contribute to variability between studies. Nevertheless, our results present a novel

207 finding regarding circulating CTRP3 levels and raises interesting questions on the factors that

208 regulate CTRP3 concentrations in human subjects. Further research is needed to clarify how

209 CTRP3 is regulated and previous studies performed with data from both genders combined should

210 be reexamined with gender treated as an independent variable. Future studies need to be performed

211 to elucidate the mechanism by which the novel adipokine CTRP3 is regulated and how gender

212 affects this regulation. Our findings support the newly released National Institutes of Health (NIH)

213 reproducibility guidelines the "NIH expects that sex as a biological variable (SABV) will be

214 considered in research designs, analyses..." (NIH 2016). 


\section{Figure legends}

218 Figure 1: Circulating CTRP3 levels were compared across the population and within gender

219 based on the: A) presence of coronary artery disease (CAD); B) tobacco use; C) hypertension; or 220 D) dyslipidemia. No differences in circulating CTRP3 levels were observed within this 221 population. Data are presented as mean \pm SEM. Significance was set at $\mathrm{p}<0.05$.

222 Figure 2: Circulating CTRP3 level by gender, obesity, and type 2 Diabetes. A) Circulating 223 CTRP3 levels were similar between all male and female subjects. B) CTRP3 levels were 224 different when examined in the presence of obesity. Specifically, CTRP3 levels were higher in 225 lean females compared with lean male subjects, but CTRP3 levels were reduced in the obese 226 females. On the other hand, CTRP3 levels were significantly higher in obese male subjects. C)

227 The diagnosis of Type 2 diabetes had no association with circulating CTRP3 levels in male 228 subjects but diabetic females had $\sim 50 \%$ lower level of circulating CTRP3 than non-diabetic 229 females. Data are presented as mean \pm SEM. Significance was set at $\mathrm{p}<0.05$. 
233 Figure 1

A

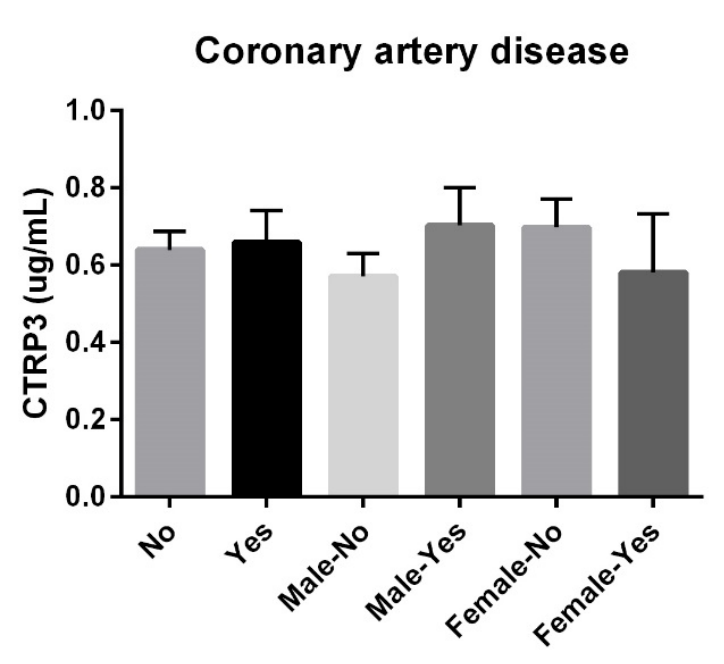

C

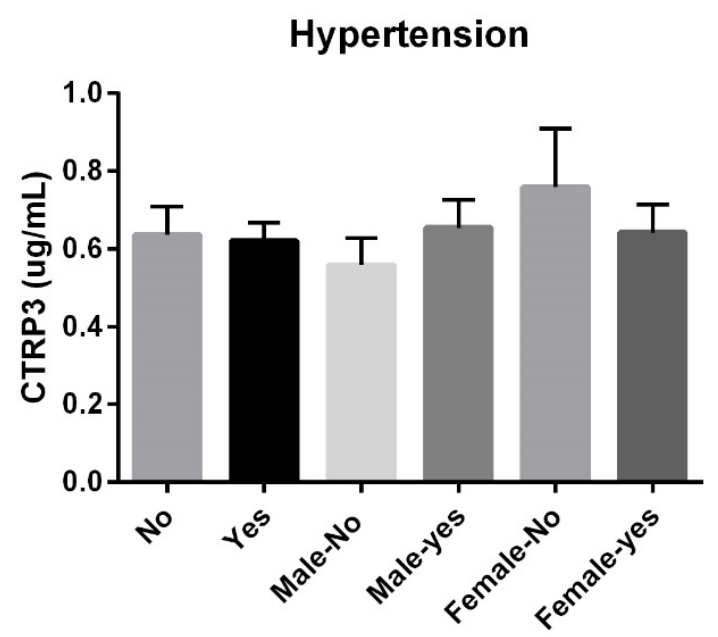

B

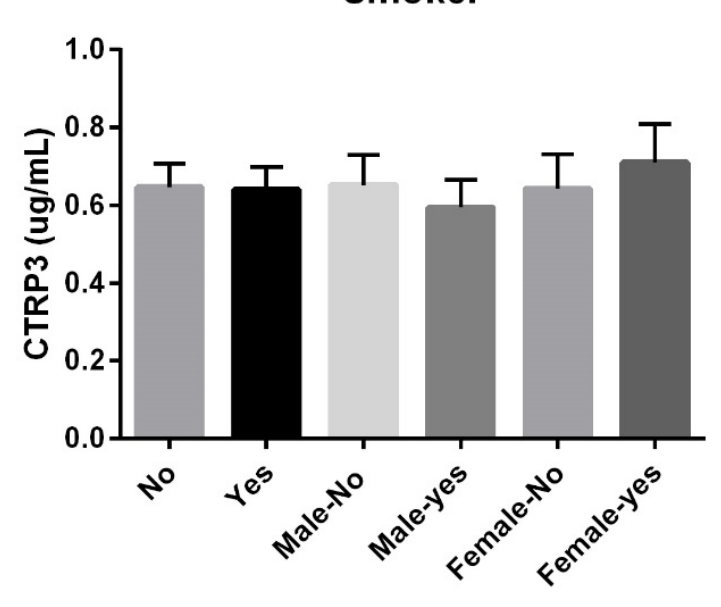

D

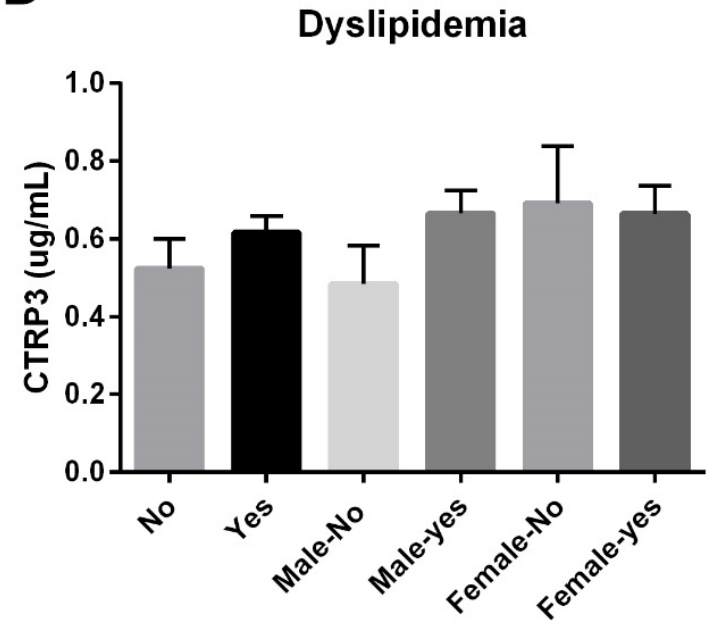


237 Figure 2

A

Gender

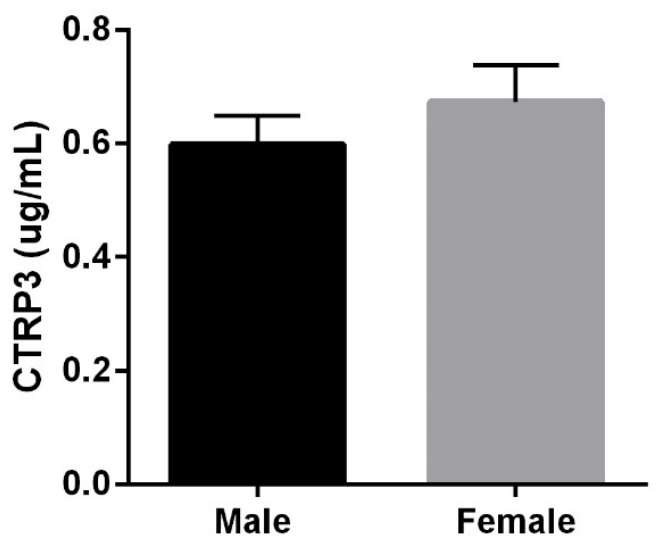

B

Obesity

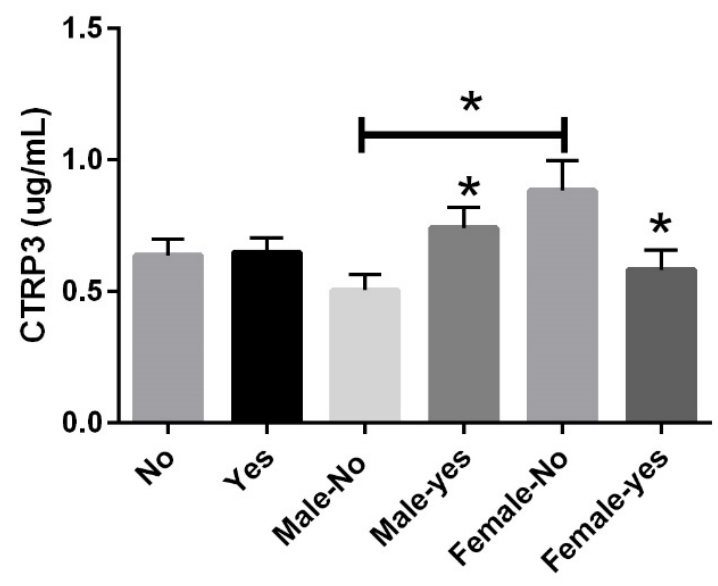

C

\section{Diabetes}

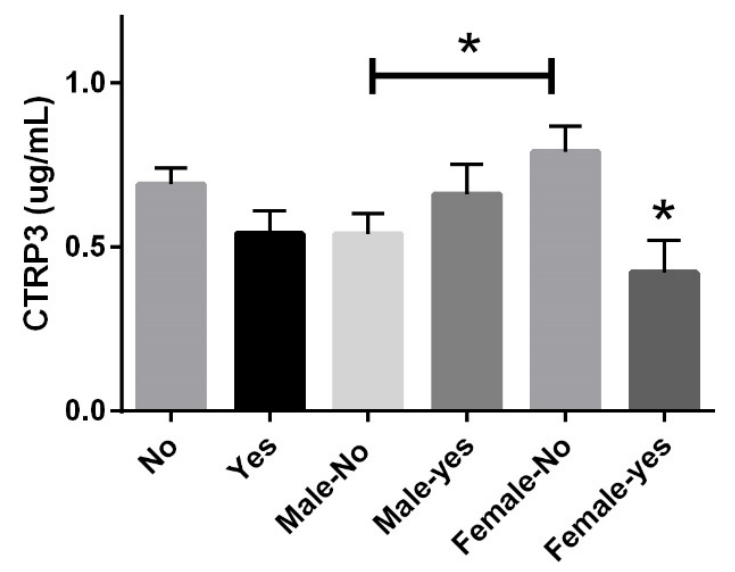


239

240

241

242

243

244

245

246

247

248

249

250

251

252

253

254

255

256

257

258

259

260

261

262

263

264

265

266

267

268

269

270

271

272

273

274

275

276

277

278

279

280

281

282

283

References

Ban B, Bai B, Zhang M, Hu J, Ramanjaneya M, Tan BK, and Chen J. 2014. Low serum cartonectin/CTRP3 concentrations in newly diagnosed type 2 diabetes mellitus: in vivo regulation of cartonectin by glucose. PLoS One 9:e112931. 10.1371/journal.pone.0112931

Ben-Menachem E. 2007. Clinical guidelines on the identification, evaluation, and treatment of overweight and obesity in adults: The evidence report. Epilepsia 48:42-45. 10.1111/j.15281167.2007.01402.x

Choi KM, Hwang SY, Hong HC, Choi HY, Yoo HJ, Youn BS, Baik SH, and Seo HS. 2014. Implications of C1q/TNF-related protein-3 (CTRP-3) and progranulin in patients with acute coronary syndrome and stable angina pectoris. Cardiovasc Diabetol 13:14. 10.1186/1475-2840-13-14

Choi KM, Hwang SY, Hong HC, Yang SJ, Choi HY, Yoo HJ, Lee KW, Nam MS, Park YS, Woo JT, Kim YS, Choi DS, Youn BS, and Baik SH. 2012. C1q/TNF-related protein-3 (CTRP-3) and pigment epitheliumderived factor (PEDF) concentrations in patients with type 2 diabetes and metabolic syndrome. Diabetes 61:2932-2936. 10.2337/db12-0217

Deng W, Li C, Zhang Y, Zhao J, Yang M, Tian M, Li L, Zheng Y, Chen B, and Yang G. 2015. Serum C1q/TNFrelated protein-3 (CTRP3) levels are decreased in obesity and hypertension and are negatively correlated with parameters of insulin resistance. Diabetol Metab Syndr 7:33. 10.1186/s13098015-0029-0

Flegal KM, Carroll MD, Kit BK, and Ogden CL. 2012. Prevalence of Obesity and Trends in the Distribution of Body Mass Index Among US Adults, 1999-2010. Jama-Journal of the American Medical Association 307:491-497. 10.1001/jama.2012.39

Garg A. 2006. Adipose tissue dysfunction in obesity and lipodystrophy. Clin Cornerstone 8 Suppl 4:S7S13.

Gaziano T, Reddy KS, Paccaud F, Horton S, and Chaturvedi V. 2006. Cardiovascular Disease. In: Jamison DT, Breman JG, Measham AR, Alleyne G, Claeson M, Evans DB, Jha P, Mills A, and Musgrove P, eds. Disease Control Priorities in Developing Countries. 2nd ed. Washington (DC).

Jankovic BD, Rakic L, Veskov R, and Horvat J. 1969. Anaphylactic reaction in the cat following intraventricular and intravenous injections of antigen. Experientia 25:864-865.

NIH. 2016. Rigor and Transparency. Available at http://grants.nih.gov/reproducibility/faqs.htm (accessed 04/02/2016 2016).

Ogden CL, Carroll MD, Kit BK, and Flegal KM. 2012. Prevalence of Obesity and Trends in Body Mass Index Among US Children and Adolescents, 1999-2010. Jama-Journal of the American Medical Association 307:483-490. 10.1001/jama.2012.40

Peterson JM, Wei Z, and Wong GW. 2010. C1q/TNF-related protein-3 (CTRP3), a novel adipokine that regulates hepatic glucose output. J Biol Chem 285:39691-39701. 10.1074/jbc.M110.180695

Qu H, Deng M, Wang H, Wei H, Liu F, Wu J, and Deng H. 2015. Plasma CTRP-3 concentrations in Chinese patients with obesity and type II diabetes negatively correlate with insulin resistance. J Clin Lipidol 9:289-294. 10.1016/j.jacl.2015.03.006

Seldin MM, Peterson JM, Byerly MS, Wei Z, and Wong GW. 2012. Myonectin (CTRP15), a novel myokine that links skeletal muscle to systemic lipid homeostasis. J Biol Chem 287:11968-11980. 10.1074/jbc.M111.336834

Seldin MM, Tan SY, and Wong GW. 2014. Metabolic function of the CTRP family of hormones. Rev Endocr Metab Disord 15:111-123. 10.1007/s11154-013-9255-7

Smith SC, Jr. 2007. Multiple risk factors for cardiovascular disease and diabetes mellitus. Am J Med 120:S3-S11. 10.1016/j.amjmed.2007.01.002 
284

285

286

287

288

289

290

291

292

293

294

295

296

297

298

299

300

301

302

303

304

305

306

307

308

309

310

311

312

313

314

315

316

317

318

319

320

321
Tan BK, Chen J, Hu J, Amar O, Mattu HS, Adya R, Patel V, Ramanjaneya M, Lehnert H, and Randeva HS. 2013. Metformin increases the novel adipokine cartonectin/CTRP3 in women with polycystic ovary syndrome. J Clin Endocrinol Metab 98:E1891-1900. 10.1210/jc.2013-2227

Wang YX, Jin JB, and Wang FZ. 1986. [Changes in transmembrane potentials of cardiac ventricular fibers during the early stage of acute cardiac ischemia and protective effects of vagal stimulation in rabbits]. Sheng Li Xue Bao 38:318-324.

Wei Z, Peterson JM, Lei X, Cebotaru L, Wolfgang MJ, Baldeviano GC, and Wong GW. 2012. C1q/TNFrelated protein-12 (CTRP12), a novel adipokine that improves insulin sensitivity and glycemic control in mouse models of obesity and diabetes. J Biol Chem 287:10301-10315. 10.1074/jbc.M111.303651

Wei Z, Peterson JM, and Wong GW. 2011. Metabolic regulation by C1q/TNF-related protein-13 (CTRP13): activation OF AMP-activated protein kinase and suppression of fatty acid-induced JNK signaling. J Biol Chem 286:15652-15665. 10.1074/jbc.M110.201087

Wei Z, Seldin MM, Natarajan N, Djemal DC, Peterson JM, and Wong GW. 2013. C1q/tumor necrosis factor-related protein 11 (CTRP11), a novel adipose stroma-derived regulator of adipogenesis. $J$ Biol Chem 288:10214-10229. 10.1074/jbc.M113.458711

Wolf RM, Steele KE, Peterson LA, Magnuson TH, Schweitzer MA, and Wong GW. 2015. Lower Circulating C1q/TNF-Related Protein-3 (CTRP3) Levels Are Associated with Obesity: A Cross-Sectional Study. PLoS One 10:e0133955. 10.1371/journal.pone.0133955

Wong GW, Krawczyk SA, Kitidis-Mitrokostas C, Ge G, Spooner E, Hug C, Gimeno R, and Lodish HF. 2009. Identification and characterization of CTRP9, a novel secreted glycoprotein, from adipose tissue that reduces serum glucose in mice and forms heterotrimers with adiponectin. FASEB J 23:241258. 10.1096/fj.08-114991

Wong GW, Krawczyk SA, Kitidis-Mitrokostas C, Revett T, Gimeno R, and Lodish HF. 2008. Molecular, biochemical and functional characterizations of C1q/TNF family members: adipose-tissueselective expression patterns, regulation by PPAR-gamma agonist, cysteine-mediated oligomerizations, combinatorial associations and metabolic functions. Biochem J 416:161-177. 10.1042/BJ20081240

Wong GW, Wang J, Hug C, Tsao TS, and Lodish HF. 2004. A family of Acrp30/adiponectin structural and functional paralogs. Proc Natl Acad Sci U S A 101:10302-10307. 10.1073/pnas.0403760101

Wu D, Lei H, Wang JY, Zhang CL, Feng H, Fu FY, Li L, and Wu LL. 2015. CTRP3 attenuates post-infarct cardiac fibrosis by targeting Smad3 activation and inhibiting myofibroblast differentiation. $\mathrm{J} \mathrm{Mol}$ Med (Berl) 93:1311-1325. 10.1007/s00109-015-1309-8

Yi W, Sun Y, Yuan Y, Lau WB, Zheng Q, Wang X, Wang Y, Shang X, Gao E, Koch WJ, and Ma XL. 2012. $\mathrm{C} 1 \mathrm{q} /$ tumor necrosis factor-related protein-3, a newly identified adipokine, is a novel antiapoptotic, proangiogenic, and cardioprotective molecule in the ischemic mouse heart. Circulation 125:3159-3169. 10.1161/CIRCULATIONAHA.112.099937 